\title{
The effects of movement velocity, mass displaced, and task certainty on associated postural adjustments made by normal and hemiplegic individuals
}

\author{
FB HORAK, P ESSELMAN, ME ANDERSON, MK LYNCH \\ From the Departments of Rehabilitation Medicine and Physiology and Biophysics, University of Washington \\ School of Medicine, Seattle, Washington, USA
}

SUMMARY The activation times for trunk and leg muscles were examined in normal and left hemiplegic subjects who raised their right arms at different velocities in self-paced or reaction time conditions. Activity in these postural muscles preceded arm displacement, and they were activated in a similar sequence during all types of rapid movements. The presence and sequencing of associated postural adjustments were more variable during slow movements.

Movement of an extremity, such as the arm, causes dynamic forces to be applied to the trunk, and these will act on the multisegmented kinematic chain between the shoulder and the base of support. The forces acting between a moving arm and the trunk are complex, but they must include linear centrifugal forces, inertial reactive forces, and torsional movements. In addition to the inertial reactions, the movement may cause a static displacement of body mass with respect to the support base, such that a postural adjustment will be necessary to maintain stability by keeping the center of mass over the vertical projection of the supporting base. If stability is to be maintained during voluntary movement, it is likely that neurally controlled postural adjustment or stabilisation must be produced by muscular activity, as a supplement to reactive forces provided by structures such as the ligaments. The required muscular activity could be controlled in two ways: (1) it could be centrally programmed, as part of the total motor act, or (2) it could be initiated reflexly by the consequences of the muscular contractions and/or the movement.

Belen'kii et al' recorded the electromyographic (EMG) activity of the anterior deltoid and several muscles in the trunk and lower extremities in standing normal individuals who rapidly raised their arm

Address for reprint requests: Dr Fay B Horak, Neurological Sciences Institute, Good Samaritan Hospital and Medical Center, 1015 NW 22nd Avenue, Portland, Oregon 97210, USA

Received 16 May 1983 and in final revised form 7 March 1984. Accepted 10 March 1984 to a horizontal position following an auditory signal. Of the muscles examined, the earliest changes in EMG activity occurred in the biceps femoris and in the lumbosacral muscles of the back. These changes in EMG activity of back and hip muscles preceded $\bigcirc$ activation of anterior deltoid, the prime mover, by $\unrhd$ as much as $70-80 \mathrm{~ms}$ and occurred more rapidly than voluntary reaction time activation of the same 8 back and hip muscles. Thus, it would appear thato this axial muscle activity must be "preprogrammed" as part of the centrally initiated motor command leading to arm movement and could not result from sensory input initiated by the arm movement or muscle activity in the prime movers at the shoulder.

If the preparatory postural muscle activity of subjects with neurological dysfunction is to be compared to the patterns seen in normal subjects, it is necessary to know more about the normal variability of these patterns and the changes that different factors might produce in preparatory muscle activity. In the present paper, we describe the effect of three factors (movement velocity, mass displaced, and prior knowledge of the movement to be made) on the timing of EMG activity in muscles acting at the shoulder, lower back, and hip in normal individuals of different ages. Because it also has been suggested that deficits in central programming of postural adjustment and stabilisation may underly the postural instability present in many patients with CNS defects resulting in hemiplegia or Parkinsonism, ${ }^{2-4}$ we also report the patterns of EMG activity in hip, trunk, and shoulder muscles of individuals with left-sided hemiplegia who were asked to raise their right arms. 
Preliminary results have been reported in abstract form. ${ }^{5}$

\section{Methods}

Subjects

Normal individuals. Data were collected from 30 normal volunteers, 15 men and 15 women, ranging in age from 20 to 82 years (mean $47 \cdot 5$ years). There were five individuals in each of the five youngest decades, and the remaining five were between the ages of 70 and 82 . All were active, many (even those in the 80th decade) engaged regularly in athletic activities, and all initially reported no neurological or musculoskeletal defects. Upon careful questioning, however, several admitted to previous fractures, sprains, or dislocations. Data from these subjects were compared carefully with those from the rest of the sample, and none stood out as remarkable with the exception of those from one woman who had experienced a right shoulder dislocation seven years prior to testing. This woman, however, also was one of the oldest subjects (age 75), and her data were included because, although they fell on the extreme of the distribution, they were not distinctly separate from data from the rest of the subjects.

Hemiplegic individuals. Data also were collected from thirteen left-sided hemiplegic volunteers ranging in age from 25 to 79 years (mean 58.5 years). The elapsed time since their strokes ranged from 2 months to 24 years. Five walked without braces and ambulation aids and were active enough to play golf or volleyball one-handed or work part time. An additional two were fairly active and did not use a wheelchair, although they did have braces, and two used canes as ambulation aids. The remaining six used a wheelchair some or all of the time. All could stand unsupported for the testing session, with rest breaks between each sequence.

\section{Testing procedure}

Subjects stood on the floor in their stocking feet or flat shoes, with approximately 6 inch $(15 \mathrm{~cm})$ separation of the heels. Right arm position (shoulder joint angle) was measured by an electrogoniometer arrangement. A long rigid aluminum tube was strapped to the arm with velcro bands just above the wrist and the elbow. This tube kept the elbow angle fixed in an extended position and accepted a thin rod that slid longitudinally within the tube. The rod was attached via a parallelogram with hinged corners to a rotary potentiometer centered on the shoulder joint. ${ }^{6}$ This arrangement allowed sagittal rotational changes in shoulder joint angle to be measured without the need for exact matching of the mechanical coupling apparatus to translational or adduction-abduction movements of the arm.

Beckman miniature silver-silver chloride disc electrodes were applied to the skin with adhesive collars positioned approximately $2 \mathrm{~cm}$ apart near the midpoint of the right anterior deltoid, right and left biceps femoris, and right and left paraspinal (primarily erector spinae) muscles at the level of the iliac crest. Electrode placement was checked by recording electromyographic (EMG) activity produced as the subject flexed the shoulder (anterior deltoid), stood upright from a flexed position at the waist (paraspinals), and flexed the knee to lift the foot, hyperextended the knee and hip with the foot planted, and externally rotated the leg at the hip (biceps femoris). Differentially-recorded EMG signals were preamplified with unity gain input stages situated near the subject, and signals were transmitted via a photocouple to variable gain amplifiers for further amplification. Amplified EMG signals, potentiometer output, and DC voltage steps indicating signal light activation (see below) were recorded on 7 channel analog FM tape.

Each normal subject was tested in four experimental paradigms. These will be designated (1) fast self-paced, (2) slow self-paced, (3) reaction time, and (4) weighted selfpaced. The instructions and subject behavior in each were as follows:

(1) Fast self-paced. The subject stood with his arms at his side and was asked to elevate his right arm in the parasagittal plane as quickly as possible to approximately horizontal, hold it there for a brief period of time, rapidly return to the starting position, and repeat this sequence until asked to stop. He was to determine his own repetition rate, but during the performance, subjects often were encouraged to sustain the start and elevated positions for longer periods of time so that steady baseline position values could be obtained and swinging movements were eliminated. Subjects also were encouraged repeatedly throughout the trials to make the movements as rapidly as possible.

(2) Slow self-paced. Subjects were asked to make arm displacements similar to those described above, but at slower and more variable movement speeds. If subjects made only very rapid or very slow movements during these trials, they were asked to slow down or speed up their movements to insure a variety of movement velocities.

(3) Reaction time. Subjects faced two lights mounted at about eye level. When the right light was illuminated, they were to raise their right arm as rapidly as possible and keep it elevated until the light was extinguished. When the left light was illuminated, they were to raise their left arm (without an attached recording device) in a similar manner. The experimenter used low acoustic noise switches to illuminate the lights in random order and at variable intervals.

(4) Weighted self-paced. A $0.9 \mathrm{~kg}$ sandbag weight was strapped to the right wrist, and the subject was asked to repeat the rapid movements described in the fast self-paced paradigm.

All requested movements were demonstrated before each test run, the subject made several practice movements, and then data were gathered for 20-30 trials during each paradigm.

Hemiplegic subjects were asked to do only the fast and weighted self-paced movements with their right, noninvolved arm, each for 15-20 trials.

\section{Data analysis}

Taped data were photographed from an oscilloscope trace onto moving kymograph film, and measurements of the duration and amplitude of the movement and time of EMG onset for each muscle relative to movement onset were measured from projected film images. Movement onset was defined as the first detectable change in resting arm position measured from the potentiometer trace (fig 1). For normal subject data, the filmed image and time and amplitude calibrations were projected onto an opaque surface. The arm of an electronic digitiser (Numonics) 
could be moved to the projected point to be measured, and the $\mathrm{x}$ and $\mathrm{y}$ coordinates of the arm at that point were stored in a computer as time and amplitude measurements, respectively. For hemiplegic subjects, the filmed data and calibrations were projected onto graph paper, and measurements were recorded manually. We would estimate that time measurement errors were within $\pm 5 \mathrm{~ms}$ for each technique. Data were measured by two different experimenters, but a comparison of the relative onset times for right deltoid and the other muscles, as measured by the two readers using the two techniques, showed a correlation coefficient of greater than $0 \cdot 9$. Any measurement error, of course, will increase as the signal to noise ratio decreases, and this was more of a problem with the slow movements and movements of the hemiplegic limbs, in which EMG activity was reduced.

\section{Statistical analysis}

Measures of central tendency (mean time of EMG onset, mean velocities) were compared under different movement conditions, between different muscles, or between normal and hemiplegic individuals using Student's $t$ test. The chi-square $\left(\chi^{2}\right)$ statistic was used to compare the frequency of muscle activation for the same muscle in fast and slow movement conditions, and linear correlations were calculated using the method of least squares to examine the relationships between velocity and muscle activation time and between the times at which deltoid and leg muscles were activated. Within subject variability of slow movement velocities was assessed using the coefficient of variability.

\section{Results}

Rapid movements made by normal subjects

When normal subjects made fast self-paced movements of the right arm without a wrist weight, postural muscle activity in the ipsilateral biceps femoris or one of the paraspinal muscles always preceded activity in the prime mover, right deltoid. A typical example is shown in the record of fig 1 , in which the

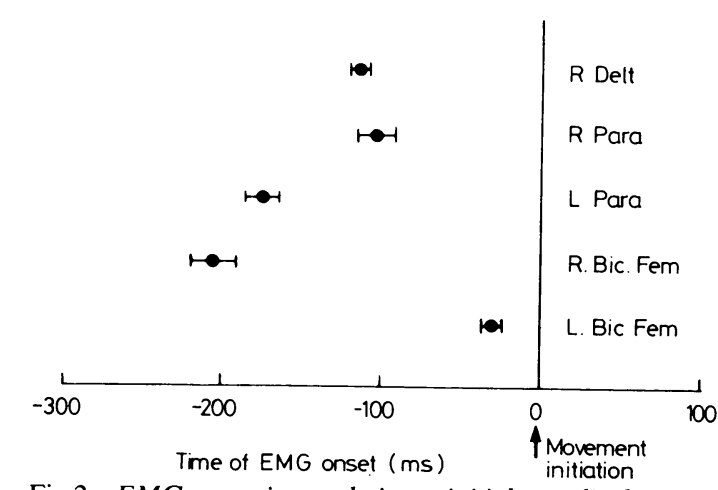

Fig 2 EMG onset times relative to initial arm displacement in the fast self-paced mode. Values are the mean $\pm S E$ for 29 normal subjects. Negative values precede movement initiation. $R$ Delt, right anterior deltoid; $R$ Para, right paraspinal muscles; L Para, left paraspinal muscles; $R$ Bic Fem, right biceps femoris; L Bic Fem, left biceps femoris.

first muscle to be activated was right biceps femoris followed by activation of left paraspinal muscles. In this subject activity in right paraspinal and left biceps femoris muscles was also initiated prior to measurable activity in right deltoid.

In most subjects $(19 / 29)$, activity started in the right biceps femoris (fig 2, 3A). In others, the paraspinals, usually on the contralateral side $(8 / 29)$, were activated first. On the average, activity in right biceps femoris preceded the onset of movement by $205 \mathrm{~ms}( \pm 15 \mathrm{~ms} \mathrm{SE})$ and preceded activity in the prime mover (right deltoid) by $90 \mathrm{~ms}( \pm 10 \mathrm{~ms} \mathrm{SE})$. Activity in the ipsilateral paraspinal (right) and right deltoid muscles usually started at about the same time. The last muscle of the five to be activated was the contralateral (left) biceps femoris for 24/29 subjects. Among the five exceptions, right paraspinal muscles were activated last in four subjects, and in one, the last muscle to be activated was the prime mover, right deltoid.

When the wrist was weighted, the sequence of EMG activation was similar to that seen during unweighted, self-paced rapid movements (fig $3 \mathrm{~A}$ and $B)$. The time between EMG initiation and arm displacement, however, was about $25 \mathrm{~ms}$ longer for all muscles than it was in the unweighted condition. To normalise for this time shift, EMG activation times for back and leg muscles shown in figure 3 and subsequent figures are expressed relative to the onset of activity in the prime mover, the right anterior deltoid.

When the rapid arm movement was made in a two choice reaction time paradigm, the sequence of EMG activity was similar to that seen in the other two types of rapid movement, in which the ipsilateral (R) biceps or contralateral (L) paraspinals was 


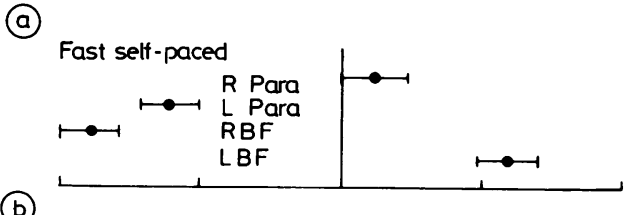

(b)

Weighted
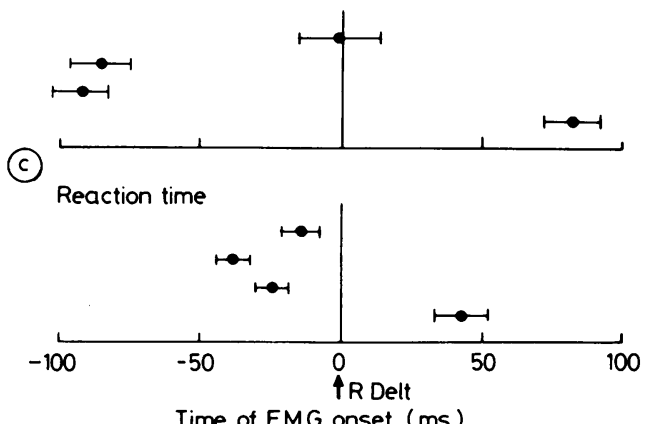

Time of EMG onset ( $\mathrm{ms}$ )

Fig 3 EMG onset times relative to activation of right anterior deltoid during rapid movements. A. Fast, self-paced mode, 29 normal subjects. $B$. Wrist weighted mode, 27 normal subjects. $C$. Choice reaction time mode, $R$ arm movements only, 28 normal subjects. All values are mean $\pm S E$. Negative values precede initiation of $E M G$ activity in right anterior deltoid. Abbreviations as in fig. 1.

activated first and contralateral (L) biceps was activated last (fig $3 \mathrm{C}$ ). Compared to fast self-paced movements, the biggest change in the reaction time condition was the more synchronous activation of muscles. In the population as a whole, the ipsilateral right biceps femoris was activated later and the contralateral left biceps femoris earlier, relative both to deltoid activity (fig 3 ) and to movement initiation. This shift of leg muscle timing occurred in 20 of the 27 individual subjects in which activity could be compared during self-paced and two choice reaction time movements.

If the left arm were to have been raised, instead of the right, the normal pattern would predict that the sequence of activation of muscles on the two sides would be reversed. When the arm to be raised was unknown (two choice reaction), initiation of biceps femoris activity in a particular leg, for example, seemed to shift to a point in time intermediate between the time it would be activated if it was consistently ipsilateral or consistently contralateral to the moving arm.

The reaction time from light onset to movement initiation varied considerably between and within subjects, yet the onset of ipsilateral leg muscle activity, which might be considered a reflection of postural preparation, remained closely related to the time of activation of the prime mover and the onset

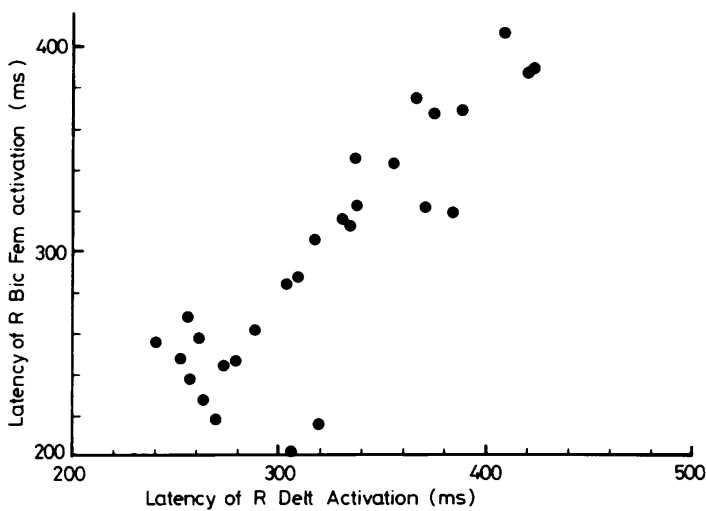

Fig 4 Relation between mean time of initial EMG activity in right anterior deltoid and right biceps femoris of normal subjects.

of displacement, instead of to the stimulus light onset. This is implied in fig $3 \mathrm{C}$, in which there is a small standard error of right biceps femoris onset time relative to right deltoid onset. It is shown more explicitly in fig 4 , in which the latency of right biceps femoris vs right deltoid EMG onset following target
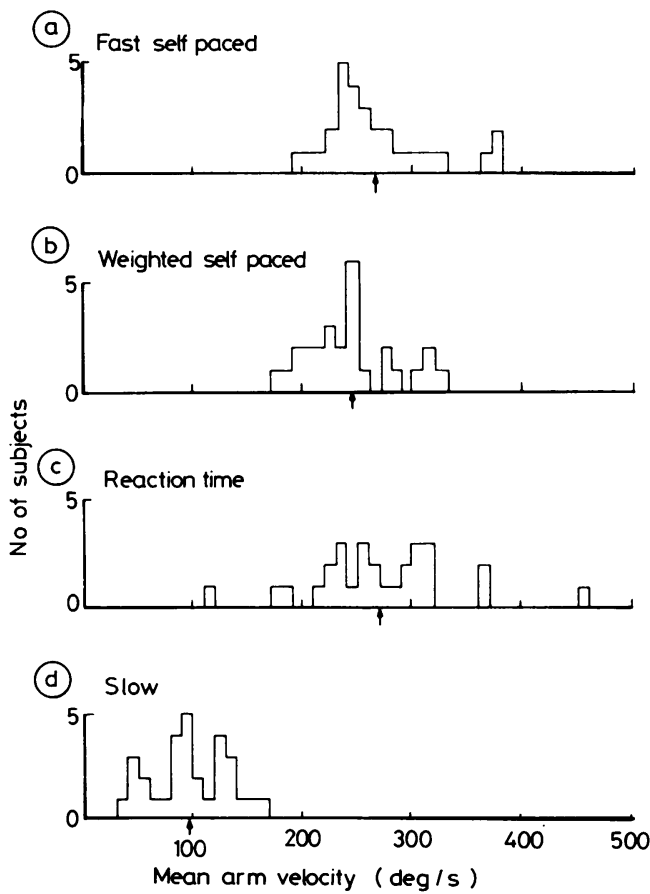

Fig 5 Mean velocity of movements made by normal subjects. $A$. Fast self-paced mode; $n=29$ subjects. $B$. Weighted self-paced; $n=27$ subjects. $C$. Reaction time; $n=$ 28 subjects. $D$. Slow self-paced; $n=30$ subjects. 
light onset is plotted for each subject. The mean time of EMG initiation in both muscles varied considerably, consistent with a wide range of mean reaction times for the various subjects, but EMG onset times in the two muscles were positively correlated with a correlation coefficient of $0.92(p<0.0001)$.

Mean velocities for movements made by each subject in the fast self-paced, weighted, and reaction time conditions are shown in fig 5, A-C. The mean velocities for the sample, as indicated by the arrows in the histograms, were 267,244 , and 270 degrees/s for fast, weighted, and reaction time conditions, respectively. Mean velocities of weighted movements were significantly slower than those of fast self-paced or reaction time movements $(t$ test, $\mathrm{p}<$ $0 \cdot 001)$. There was no significant difference, however, between movement velocities in the fast selfpaced and reaction time modes, although the variability of velocities of reaction time movements was greater.

Slow, self-paced movements made by normal subjects

When subjects were asked to raise their right arms slowly, the mean velocity of movements made by all subjects $(97.9 \mathrm{deg} / \mathrm{s} \pm 6.5 \mathrm{deg} / \mathrm{s} \mathrm{SE})$ was less than half the velocity of the fast, self-paced movements ( $267 \mathrm{deg} / \mathrm{s}$, fig 5D). Under these conditions, the timing, and often the sequencing, of activity in the various muscles changed from those seen during rapid movements.

Although the initial measurable EMG activity during slow movements most often was in ipsilateral

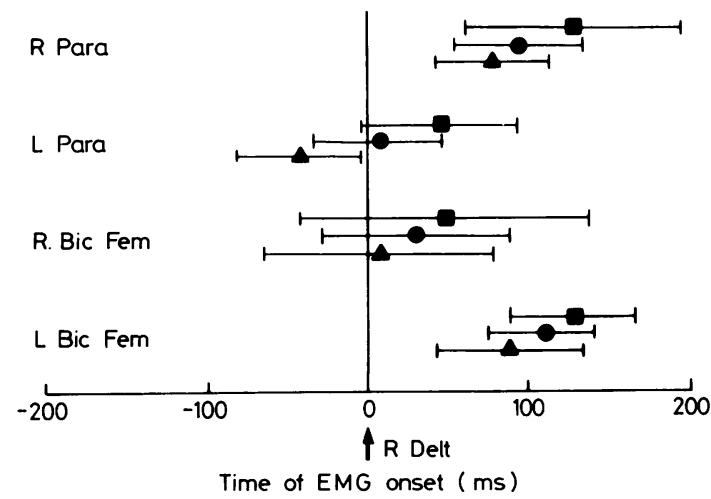

Fig 6 EMG onset times relative to activation of right anterior deltoid during slow movements made by normal subjects. Mean $\pm S E$ of onset times are shown for all slow movements (๑), for movements with mean velocities $\leqslant 100 \mathrm{deg} / \mathrm{s}$. (口), and for movements with mean velocities $>100 \mathrm{deg} / \mathrm{s}$ (4). Negative values precede initiation of EMG activity in right anterior deltoid. Abbreviations as in fig 1 . (right) biceps femoris (11/30 subjects) or one of the paraspinal muscles (ipsilateral in $2 / 30$ subjects, contralateral in $8 / 30$ subjects), in nine of the 30 subjects, deltoid showed the earliest measurable activity. When expressed relative to right deltoid activation, activity in right biceps femoris and the paraspinal muscles on both sides was initiated later during slow movements than during rapid self-paced movements (compare fig 6 , circles, with fig $3 \mathrm{~A}$ ). There also was considerably more variation in the time at which individual muscles were activated, and as a result, the mean onset times for the ipsilateral (right) biceps femoris and contralateral (left) paraspinals for the entire sample were later than that of right deltoid (fig 6), even though one of the former two muscle groups was activated before deltoid in most individual subjects. The right biceps femoris, especially, showed considerable variation in time of activation and actually was the last muscle to be activated in eight of the $\mathbf{3 0}$ subjects. This is in contrast to the muscle activation sequence for fast selfpaced movements, in which this muscle never had the latest mean onset time in any of the subjects tested.

During slow self-paced movements, some back and leg muscles never showed measurable activity, or the activity appeared in fewer trials than it did $\underset{\mathbb{\Phi}}{\stackrel{\rho}{+}}$ during rapid movement trials. For example, the mean probability with which EMG activity was recorded in right biceps femoris for all subjects dropped from 0.93 during fast self-paced movements to 0.67 during slow movements. For the left biceps femoris EMG, the drop was from 0.98 (fast) to 0.70 (slow). This change was significant at the 0.05 level for both leg muscles $\left(\chi^{2}\right)$. For paraspinal muscles, there was only a small, insignificant trend toward a reduced incidence of measurable activity during slow movements, compared to rapid ones.

If subjects are divided into those with mean slow movement velocities less than $100 \mathrm{deg} / \mathrm{s}$ and those greater or equal to $100 \mathrm{deg} / \mathrm{s}$ (fig 6, squares and triangles, respectively), then the mean EMG onset times (relative to deltoid activity) were consistently earliest in those subjects with higher mean velocities (triangles).

The trial-to-trial variability in velocity of slow movements made by individual subjects also was greater than it was for fast self-paced movements. The mean coefficient of variability was 0.11 for the velocities of fast, self-paced and 0.33 for the velocities of slow self-paced movements. The variability in the velocity of movements made by each subject allowed us to examine the correlation between movement velocity on individual trials and the onset times of the muscles studied. In many individual subjects, there also was a statistically significant 
inverse correlation between the time of muscle activation prior to movement onset and the velocity of the associated movement. This tendency for muscles to become activated earlier with higher velocity movements was most often significant for the prime mover, right deltoid ( 16 of 30 subjects; $\mathrm{p}<0.05$, r $=-0.430$ to -0.874$)$. For the other muscles, when measurable activity was present in more than five of the slow movement trials, the correlation between movement velocity and the lead time at which a measurable increase in EMG activity could be detected was significant $(p<0.05)$ for right paraspinals in eight of 25 subjects, for left paraspinals in 11 of 22 subjects, for right biceps femoris in eight of 19 subjects, and for left biceps femoris in 10 of 20 subjects.

Relationships between movement parameters and age To allow future comparison of movement variables between normal subjects and patient populations with restricted age distributions, we examined the relationship between age and the movement and muscle timing variables measured.

Figure 7 shows mean movement velocity plotted as a function of age for each of the normal subjects studied in the fast self-paced, reaction time, weighted, and slow self-paced conditions. There was a tendency for the velocity to be inversely correlated with age in the fast and weighted self-paced conditions, but this was statistically significant only for weighted movements $(r=0.49, p<0.01)$. The lack of a significant correlation in the fast self-paced mode is, however, largely the result of the rapid movements made by the $82 \mathrm{yr}$-old-subject, and if

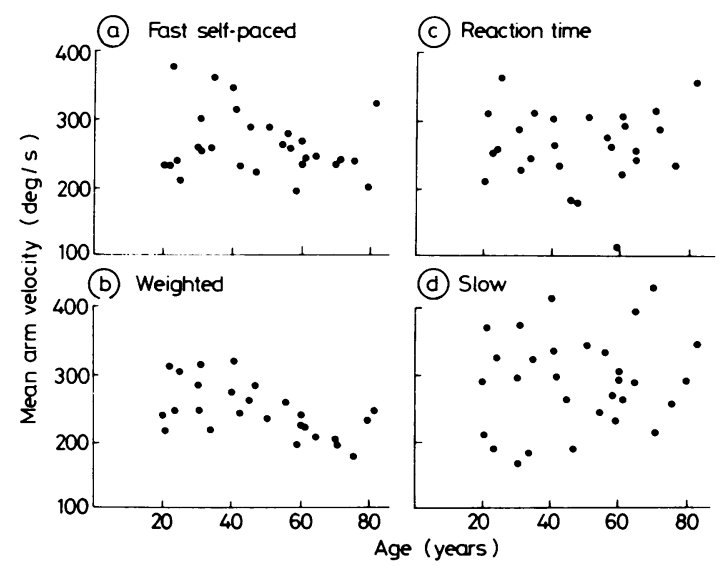

Fig 7 Relation between mean arm velocity and age for normal subjects studied during fast self-paced $(A)$, wrist weighted $(B)$, reaction time $(C)$, and slow self-paced movements (D). one compares mean velocities for subjects under and over 50 years of age, the velocities are significantly slower for the older subjects during both the fast self-paced and the weighted self-paced movements $(t$ test, $\mathrm{p}<0 \cdot 001)$. Movement velocities for subjects under and over 50 years of age were not significantly different, however, for movements made in the reaction time or slow paradigms.

Older subjects did tend to initiate movements after a longer delay in the reaction time paradigm, as shown by a statistically significant correlation between age and reaction time $(r=+0.33, p<0.05)$.

In spite of the significant interaction between age and movement velocity and the weak interaction between age and reaction time, there were no significant correlations between age and the time relative to deltoid activity at which other individual muscles were activated in any of the movement conditions studied.

\section{Rapid movements made by hemiplegic subjects}

Many movements made with the right arm by left hemiplegic subjects, even in the fast self-paced or weighted paradigms, were much slower than those made by normal subjects. For example, the durations of fast, self-paced movements ranged from 198 to $622 \mathrm{~ms}$ for normal subjects, whereas they ranged from 355 to $1256 \mathrm{~ms}$ for hemiplegic individuals. To allow comparisons between EMG patterns associated with rapid movements made by the two groups of subjects, only those fast self-paced and weighted movements with durations less than $800 \mathrm{~ms}$ were analyzed quantitatively for hemiplegic subjects.

The mean times of initial EMG activation, expressed relative to right deltoid onset time, are represented by the circles in fig 8 for the four back and leg muscles. The sequences of muscle activation in both the fast self-paced and weighted self-paced modes were similar to those seen in normal subjects, although the latency of postural muscle activity on the hemiplegic side relative to deltoid activity was later than in normal subjects (triangles in fig 8). Right biceps femoris was the first muscle activated in all hemiplegic subjects studied, both during fast self-paced and weighted movements. In these 13 hemiplegic subjects, activation of right biceps femoris preceded right deltoid activity by $106 \cdot 2 \mathrm{~ms}$ $( \pm 11.0 \mathrm{~ms} \mathrm{SE})$ in the fast self-paced mode and by $74.0 \mathrm{~ms}( \pm 15.6 \mathrm{~ms} \mathrm{SE})$ in the weighted mode. These values were not significantly different from those measured for normal subjects.

The paraspinal muscles on the left (hemiplegic) side were activated next after right biceps femoris in nine of the 13 hemiplegic subjects, at an average of $47.7 \mathrm{~ms}( \pm 12.2 \mathrm{~ms} \mathrm{SE})$ prior to right deltoid activation during fast self-paced movements and $13.2 \mathrm{~ms}$ 


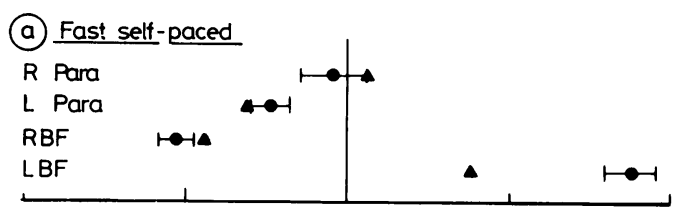

(b) Weighted

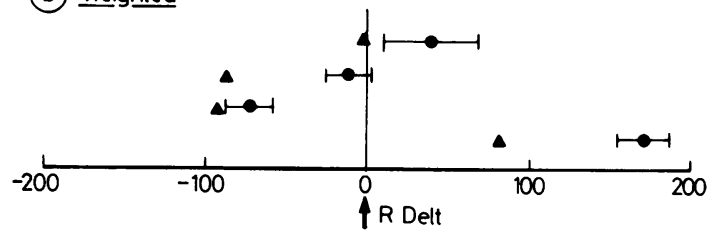

Time of EMG onset ( $\mathrm{ms}$ )

Fig $8 \quad$ EMG onset times relative to activation of right anterior deltoid for left hemiplegic subjects (circles) compared to normal individuals (triangles) taken from fig $3 A$ and $B$. A. Fast self-paced mode; $n=13$. B. Weighted mode; $n=8$. All values for hemiplegic subjects are means \pm $S E$. Negative values precede initiation of EMG activity in right anterior deltoid.

( $\pm 13.7 \mathrm{~ms} \mathrm{SE}$ ) before right deltoid activation during weighted movements. These contralateral paraspinal muscles were activated well in advance of right deltoid in both normal and hemiplegic subjects, in the fast self-paced condition, but there was no significant difference in the activation time between the two groups of subjects. For weighted movements, however, left paraspinal activity was measured later in hemiplegic than in normal subjects $(p<0.001)$.

As was the case for normal subjects, the ipsilateral (right) paraspinal muscles were activated at about the same time as the right deltoid in the fast selfpaced condition. The slightly earlier onset in hemiplegics, compared to normals, during fast self-paced movements (compare fig 8A and $3 \mathrm{~A}$ ) was not significant at the 0.05 level, and there also was no significant difference between the time of right paraspinal activation during weighted movements.

As in most normal subjects, the left (hemiplegic) biceps femoris was the last muscle to show measurable activity in 11 of 13 hemiplegic subjects studied during fast self-paced movements and in all of the eight subjects studied during weighted movements. In both conditions, the onset of measurable activity occurred later in hemiplegic than in normal individuals (compare circles and triangles in fig $8 \mathrm{~A}$ and B), $p<0.001)$. Measurable left biceps femoris activity during fast, self-paced movements of the right arm was initiated at a mean of $104 \mathrm{~ms}$ after movement initiation in left hemiplegic individuals, whereas in normal individuals, left biceps femoris activity was initiated $37 \mathrm{~ms}$ prior to movement onset (fig 2). If the function of the left biceps femoris activity was to provide hip extensor activity necessary for support when the trunk weight was shifted diagonally away from the raised right arm, then it might be predicted that the time of left biceps femoris activation would have a closer relationship to the time at which arm elevation was completed than it would to the time at which it was initiated. This, in fact, was the case. On the average, left biceps femoris activity was initiated $443 \mathrm{~ms}$ prior to termination of the fast self-paced right arm movements made by hemiplegic subjects and $426 \mathrm{~ms}$ prior to termination of fast self-paced movements made by normal subjects.

\section{Discussion}

The results of the present study indicate that when freestanding individuals raise one arm as rapidly as possible, they use essentially the same sequence of hip, back, and shoulder muscle activation, whether the movement is self-initiated or visually triggered, and regardless of the load, their advance knowledge of which arm is to be raised, or the presence of contralateral hemiplegia consequent to a cerebral vascular accident. When they raise the arm more slowly, the sequence of muscle activation becomes more variable, and some muscles with early activity prior to rapid movement show no measurable activity at all.

Other investigators have examined the activity of leg and, in some cases, back muscles during simple reaction time tasks requiring unilateral elevation of the same arm following either an auditory' or a visual stimulus. ${ }^{89}$ All of these groups found essentially the same sequence of activity in back and hip extensor muscles as was seen during any of the rapid movements in the present study. The lead time of ipsilateral hip muscle activation relative to activation of the prime mover varied, however, during rapid movements made under different conditions. In the simple reaction time condition, in which the same arm was raised on each trial and, as a consequence, there was temporal, but no spatial uncertainty about the movement to be made, ipsilateral hip extensor activity led deltoid activity by about $40-55$ ms (biceps femoris, Belen'kii et al ${ }^{1}$ 'semitendinosus, Bouisset and Zattara ${ }^{8}$ ) although in the report by Lee, ${ }^{9}$ this timing difference was not statistically significant. These values during non-choice reaction time trials were intermediate between the biceps femoris lead time $(93 \mathrm{~ms})$ measured in the fast selfpaced condition of the present study, in which there was neither spatial nor temporal uncertainty, and the lead time $(23 \mathrm{~ms})$ of the same muscle in the two choice reaction time condition, in which both tem- 
poral and spatial uncertainty existed. Thus, although spatial and temporal uncertainty did not change the sequential pattern of muscle activation, they did change the timing of postural muscle activity.

There was a strong correlation between the reaction time onset of EMG activity in the prime mover (right deltoid) and in the muscle (right biceps femoris) with the earliest associated postural adjustment activity, indicating that the early hip muscle activity was more closely related to the movement initiation than to the stimulus light onset. Cordo and Nashner ${ }^{10}$ reported only a non-significant trend for this relationship, but this may have been due to their rather small sample size.

The relative timing of EMG activity was much more sensitive to the mean velocity of the movement than it was to the mass displaced. This is perhaps not surprising, since if the preparatory EMG activity serves to help compensate for the maximum disturbing forces due to inertial and torsional moments, compensation must be maximum between the maximum acceleration and maximum velocity phases of the movement, irrespective of the mass displaced. If we had been able to quantify the amplitude of EMG activity or the force it produced, it is probable that these would have varied more with mass displaced than did the timing of EMG onset.

The major measured variable that influenced both the timing of measurable EMG activity and the probability of its occurrence in back and leg muscles was the velocity of the ensuing movement. It is true that the lower magnitude of EMG activity during slow movements decreased the signal to noise ratio and would have delayed the time at which EMG activity was detectable and, hence, increased the timing measurement error. The amplitude of EMG activity in anterior deltoid also was decreased, however, and since timing was expressed relative to right deltoid activity, it is unlikely that measurement errors could completely explain the results. It is more probable that, since less stabilisation force is necessary during low acceleration and low velocity movements, this aspect of preparatory muscle activity simply is not programmed as rigidly in association with slow movements. The relation between movement velocity and EMG onset times is important to consider in studies of associated postural adjustments made by aged or neurologically impaired subjects, some of whom may make movements at slower velocities. Changes in associated postural adjustments may be related to the velocity of the movements, and not to the age or pathology, per se.

In addition to the influence of acceleration and velocity on early preparatory. EMG activity, the sub- ject's initial postural stability also affects this preparatory activity. Cordo and Nashner ${ }^{10}$ found that the early changes in EMG activity recorded in leg muscles prior to rapid, self-initiated displacement or sound-triggered push or pull movements of the arm were markedly decreased in amplitude if the subject was supported at the shoulder or even touched a guard rail with a single finger.

Because these early associated postural adjustments precede any displacement, or even any contraction of the prime movers, it is hypothesized that they are centrally generated and are not reflexive in nature..$^{14910}$ Since damage to CNS structures could interrupt the ability to generate this normal pattern of associated postural adjustments, it is of interest to examine the activity of muscles on a hemiparetic or hemiplegic side of the body when the contralateral (normal) arm is activated. In our left hemiplegic subjects, the sequence of activity in the five muscles studied was the same as that seen in normal subjects, both in the weighted and unweighted conditions. Measurable activity on the contralateral hemiparetic side, both at the waist and the hip, occurred at a later time than it did in normal individuals, especially during weighted movements. This occurred despite the fact that the patients displayed varying amounts of spasticity on the hemiplegic side. The relative latencies of muscle activity on the nonhemiplegic side (ipsilateral to the raised arm) were normal. Pal' tsev and El' ner ${ }^{3}$ reported that if subjects with frontal lobe damage raised their paretic arm, activity in the ipsilateral (hemiparetic) biceps femoris also occurred later than it did in normals, but they did not present enough data to determine whether its activation was delayed when it was contralateral to the raised normal arm.

The delay in preparatory postural activity on the hemiplegic side during self paced movements was more striking in the left biceps femoris than in the left paraspinal muscles (fig 8). Although greater bilateral innervation of trunk muscles than leg muscles may account for this difference, any possible sparing of innervation was inadequate to prevent statistically significant delays in left paraspinal onset under conditions of increased load.

Although our hemiplegic subjects raised their arms more slowly than normal subjects when asked to move as rapidly as possible, the pattern of preparatory postural activity in hemiplegic subjects does not resemble the patterns seen in normal subjects moving their arms at slower velocities (compare figs $6 \& 8 \mathrm{~A}$ ). When normal subjects raised their arms slowly, right paraspinal and right biceps femoris activity was usually initiated after right deltoid. In contrast, the hemiplegic subjects demonstrated a sequence in the activity of leg and back 
muscles on the non-hemiplegic (right) side that was similar to that seen in the faster moving normal subjects. In fact, we may speculate that preparatory postural muscle activity on the non-hemiplegic side was utilised by hemiplegic subjects at slower arm velocities in order to compensate for their more precarious stance.

Thus, any preprogrammed descending signal that would determine the bilateral sequence of muscle activation seemed to be able to reach the cord. Whether such signals descend on separate sides of the brainstem or whether they descend on one side and distribute bilaterally at the lower brainstem or cord is not known, although associated postural responses will occur bilaterally in cats after motor cortex lesions on one side. ${ }^{11}$ Either might have been the case in our hemiplegic subjects, since the later activation of muscles on the hemiparetic side could have resulted either because information descending on the undamaged side reached a less excitable contralateral motoneuronal pool or because the number of undamaged descending fibres on the side of the injury simply was insufficient to activate motorneurons on the hemiplegic side quite as early as was the case for normal subjects. Irrespective of whether the delay in timing is determined at a high or a low level, it is possible that hemiplegic patients were not capable of making movements of the "uninvolved" arm as rapidly as normal because of delays in anticipatory activity of contralateral hemiplegic muscles important in postural stabilisation.

Early associated postural adjustments presumably provide stability for the ensuing movement. Of the variables examined in this study, the velocity of the movement to be made appeared to be most significant in determining the presence and timing of activity in the postural muscles examined.

It is a pleasure to acknowledge the technical assistance of Mark Guthrie and Beverly Bedell.
This study was supported in part by Research Grant \#G008200020 from the National Institute of Handicapped Research, Department of Education, Washington, DC 20202.

\section{References}

' Belen'kii VY, Gurfinkel VS, Pal'tsev YI. Elements of control of voluntary movements. Biophysics 1967;12:135-41.

${ }^{2}$ Martin JP. The Basal Ganglia and Posture. Philadelphia: Lippincott 1967.

${ }^{3}$ Pal' tsev YI, El'ner AM. Preparatory and compensatory period during voluntary movement in patients with involvement of the brain of different localization. Biophysics, 1967;12:161-8.

${ }^{4}$ Traub MM, Rothwell JC, Marsden CD. Anticipatory postural reflexes in Parkinson's disease and other akinetic-rigid syndromes and in cerebellar ataxia. Brain 1980;103:393-412.

${ }^{5}$ Horak FB, Anderson ME. Preparatory postural activity associated with movement. Physical Therapy 1980;60:580.

${ }^{6}$ Lamorux LW. Kinematic measurements in the study of human walking. Bulletin of Prosthetic Research 1971;10:3-84.

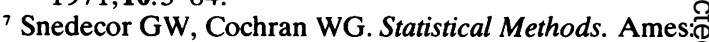
The Iowa State Press 1967.

${ }^{8}$ Bouisset S, Zattara M. A sequence of postural move ments precedes voluntary movement. Neuroscience Letters 1967;12:135-41.

${ }^{9}$ Lee WA. Anticipatory control of postural and task muscles during rapid arm flexion. Journal of Motor Behavior 1980;12:185-96.

${ }^{10}$ Cordo PJ, Nashner LM. Properties of postural adjustment associated with rapid arm movements. $J$ Neurophysiol 1982;47:287-302.

"Massion J. Role of motor cortex in postural adjustments associated with movement. In: Asanuma $\mathrm{H}$ and Wilson $\mathrm{VJ}$, eds. Integration in the nervous system. Tokyo-New York: Igaku-Shoin, 1979. 